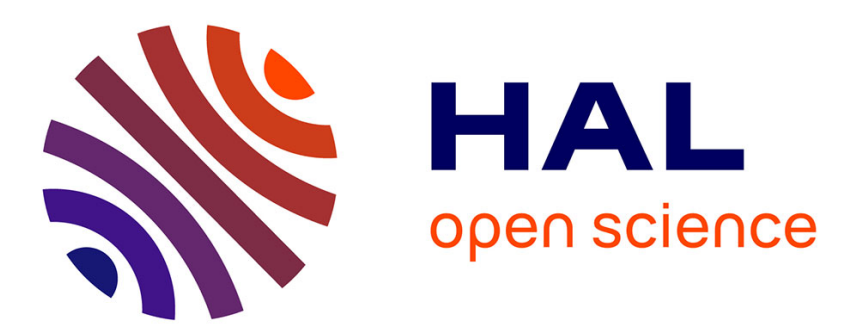

\title{
Switchable broadband light reflection in polymer-stabilized cholesteric liquid crystals
}

Corinne Binet, Michel Mitov, Monique Mauzac

\section{To cite this version:}

Corinne Binet, Michel Mitov, Monique Mauzac. Switchable broadband light reflection in polymerstabilized cholesteric liquid crystals. Journal of Applied Physics, 2001, 90 (4), pp.1730-1734. 10.1063/1.1388172 . hal-03588737

\section{HAL Id: hal-03588737 https://hal.science/hal-03588737}

Submitted on 3 Mar 2022

HAL is a multi-disciplinary open access archive for the deposit and dissemination of scientific research documents, whether they are published or not. The documents may come from teaching and research institutions in France or abroad, or from public or private research centers.
L'archive ouverte pluridisciplinaire HAL, est destinée au dépôt et à la diffusion de documents scientifiques de niveau recherche, publiés ou non, émanant des établissements d'enseignement et de recherche français ou étrangers, des laboratoires publics ou privés. 


\title{
Switchable broadband light reflection in Polymer-Stabilized Cholesteric Liquid Crystals
}

\author{
Corinne Binet, Michel Mitov a and Monique Mauzac*
}

Centre d'Elaboration de Matériaux et d'Etudes Structurales, CEMES-CNRS ${ }^{b}$, BP 4347, 31055 Toulouse cx 4, France

*Laboratoire des Interactions Moléculaires et Réactivité Chimique et Photochimique, CNRS $^{c}$, Université Paul-Sabatier, 118 route de Narbonne, 31062 Toulouse cx 4, France

Due to the existence of a macroscopic helical structure, oriented cholesteric liquid crystals (CLCs) can selectively reflect light. The wavelength bandwidth for these Bragg reflections is limited: typically a few tens of nanometers due to limited birefringence values available in organic mesomorphic compounds. Here we show that larger bandwidthes may be reached by associating two layers of Polymer-Stabilized CLCs (PSCLCs). In the case of near-IR spectrum, it is shown that the optical properties of the bilayer system are not the sum of the individual properties. Time stability is investigated because the very existence of an interdiffusion between soft matter layers. Optical characteristics of the new PSCLC can be tuned by an electric field and transitions between broadband reflecting, scattering and transparent states are possible. For applications, switchable broadband reflections in LC media are relevant for whiteor-black polarizer-free flat displays and 'smart' windows in buildings.

aCorresponding author, mitov@cemes.fr

bUPR 8011

'UMR 5623

Article history: Received 27 October 2000; accepted for publication 1 June 2001.

https://doi.org/10.1063/1.1388172 


\section{INTRODUCTION}

Cholesteric liquid crystals (CLCs) exhibit many remarkable properties due to the existence of a macroscopic helical structure [1,2]. Iridescent selective reflection colors are displayed by an uniformly oriented Grandjean planar texture, i.e. when the helical axis is perpendicular to the observation plane. Inside a small wavelength band $\Delta \lambda$, centred around $\lambda_{\mathrm{o}}$, an incident light beam parallel to the helical axis is split into two circularly polarized components: one of which is simply transmitted whereas the other is totally reflected. The rotation of the reflected circularly polarized light agrees with the screw sense of the structure. At normal incidence, maximum reflection occurs when the incident wavelength $\lambda$ matches the following Bragg law:

$\lambda=\lambda_{\mathrm{o}}=\mathrm{nxp}$,

where $\mathrm{n}=\left(\mathrm{n}_{0}+\mathrm{n}_{\mathrm{e}}\right) / 2$ is the average of the ordinary $\left(\mathrm{n}_{\mathrm{o}}\right)$ and extraordinary $\left(\mathrm{n}_{\mathrm{e}}\right)$ refractive indices of the locally uniaxial structure and $\mathrm{p}$ is the helical pitch. It is in the region $\Delta \lambda$ of the pitch band that most of optical investigations of CLCs have concentrated. $\Delta \lambda$ is given by the following relation:

$\Delta \lambda=\mathrm{p} \times \Delta \mathrm{n}$

where $\Delta \mathrm{n}=\mathrm{n}_{\mathrm{e}}-\mathrm{n}_{\mathrm{o}}$ is the birefringence. An incident beam with a wavelength out of $\Delta \lambda$ is simply transmitted.

However huge the choice for pitch amplitude is, $\Delta \mathrm{n}$ is typically limited to values below 0.3 for colorless organic materials. As a consequence, $\Delta \lambda$ is commonly less than $100 \mathrm{~nm}$ in the visible spectrum and is more often equal to $50 \mathrm{~nm}$. The selectivity is desired for several families of applications - optical filters, thermal imaging, laser or paint technologies -, but a drawback lies in the fact that the known CLC filters have a reflection bandwidth that is too limited for specific purposes, like full-color or 
white-or-black reflective displays, broadband circular polarizers or 'smart' windows in buildings where a control over the solar spectrum is desirable.

The goal of this paper is to present a phenomenon of bandwidth broadening for light reflections in Polymer-Stabilized Cholesteric Liquid Crystals (PSCLCs) with the following characteristics: (i) light reflection occurs inside a band greater than that usually expected in the wavelength range of the present study (near-IR spectrum) and is of the order of several hundreds of nanometers; (ii) in addition, the material is switchable when subjected to an electric field. Field-induced transitions between broadband reflecting, scattering and transparent states are stated.

\section{STATE OF THE ART}

We will distinguish two cases: (i) Non-switchable CLCs: the material is solid; it is a crosslinked polymer or a glass. Most of studies deal with that situation; (ii) Switchable CLCs: the major component of the material is a low molar mass LC like PSCLCs in which the LC molecules are not chemically bound to the macromolecular network - and the material can be addressed by an electric field.

\section{A. Non-switchable CLCs}

Most of studies deal with a CLC polymer network with a pitch gradient in the visible spectrum that requires an illumination gradient during a UV-crosslinking reaction [3-7]. As a consequence, $\Delta \lambda$ is greater than $300 \mathrm{~nm}$.

In the pioneering work done by Philips group [3,4], the pitch gradient is due to a photo-induced diffusion during an UV-polymerization reaction occurring in a chiral 
and nematic monomers blend with different UV-reactivities. An absorbing dye is required to create a UV-intensity gradient over the film thickness. Such broadband reflectors greatly improve the light yield (by $40 \%$ in [5]) and the energy efficiency of LC devices by recycling wrongly-polarized light in the backlight system $[5,6]$.

The broadening of the reflection band and the enhancement of polarized backlight output have been studied for two types of CLC films [8-13]: (i) a stack of red-, greenand blue-reflecting films [8-10]; and (ii) a single layer [11-13] 'that achieves its wide selective reflection band because of its engineered pitch distribution' [12].

We experienced an alternative route for increasing $\Delta \lambda$ which led to a novel broadband CLC with a pitch gradient $[14,15]$. By contrast to previous studies, the process depends on a thermal diffusion between two CLC oligomer films with different chiralities and the pitch gradient is the consequence of a concentration gradient which is then frozen by quenching. Neither polymerization reaction nor photo-induced phase separation is involved. Besides, the reflector is a single-layer CLC film in the glassy state. $\Delta \lambda$ may simply be tuned by varying the annealing time and different optical properties (like tunable bandwidths) are available [16]. The pitch gradient was demonstrated and evaluated by transmission electron microscopy investigations of cross-sections [17]. The very glassy state of the reflector was discussed as offerring new opportunities for optical data storage. This material design has then been adapted from the case of glasses to photocrosslinked networks in which a UV-curing hardens the material and irreversibly locks the helical pitch [7, 18].

Spatially modulated photoracemization in glassy chiral nematic films can also generate a pitch gradient in a CLC structure [19]. 
In the case of CLC-polymer composites with high concentrations in polymer, a novel approach that lies in a thermally-induced pitch variation simultaneously carried out with the UV-crosslinking reaction has recently been experimented [20].

\section{B. Switchable CLCs}

Studies in PSLCs correlate the influence of the chemical structure of one or several compounds in the mixture to the $\Delta \lambda$ increase [21-23]. The fact of combining chiral tolane mesogenic side-on additives into the blend shows an improvement in the birefringence and consequently increases $\Delta \lambda$ [21]. Due to a greater chiral concentration in the vicinity of the polymer network than in the bulk of a PSCLC with specific chiral additives, a distribution of two areas with different pitches is evoked to explain a reflection broadening (overlap in the reflection spectra from these two areas) [22]. The position and stereochemical configuration of the chiral centers in the side group of functionalized polymers, with respect to the backbone, play an integral role in the observed reflection spectra [23].

$\Delta \lambda$ can also be increased in PSCLCs when compounds referred to as excited-state quenchers are added to the monomeric mixtures [24]. Such quenchers work by transferring the energy of the excited state of the initiator to themselves and by absorbing UV-light. A phase separation leading to concentration fluctuations corresponding with different pitches is expected.

Here we present new PSCLCs with broadband light reflections as a result of an adaptation of our previous process using CLC glasses [15-17]. The basic idea lies in the association of two individual PSCLC layers the interdiffusion of which is 
controlled by UV-curing [25]. At a fundamental viewpoint, this situation gives the opportunity to investigate novel aspects of soft matter interfaces when borders between two chiral LC materials are mobile, diffuse and active. It is shown that the optical properties of the bilayer system are not entirely the sum of the individual properties. Additionally, for potential applications, a single sandwich-cell is preferable to a stack of two or several sandwich-cells due to absorption by glass substrates and undesired reflections at interfaces.

At each experimental step, the spectral behavior was analyzed by unpolarized light spectrophotometry. Stability of systems with time was investigated. Finally, it was shown that the optical characteristics can be modified by an electric field.

\section{EXPERIMENTAL}

Blends of a photocrosslinkable CLC substance RMB with a low molar mass CLC mixture BL090 (from Merck Ltd.) and a cholesteric oligomer SB are made. RMB is a modified siloxane compound suited for the production of optical filters [26] and consists of reactive groups and mesogenic side-groups. For each blend, 2 wt \% (compared to RMB wt.) of photoinitiator Irgacure 907 (from Ciba-Geigy) are added for crosslinking purposes. SB molecule is a siloxane cyclic chain with two types of side-chains attached to the main chain via spacers: a non-chiral mesogen and a chiral one $[26,16]$; the molar percentage of chiral mesogens in SB molecule is 50\%. BL090 has a positive dielectric anisotropy of about 20.6. LC materials are not chosen with peculiarly large values of birefringence since the goal is to enlarge the reflection bandwidth through the material design. 
Two mixtures are prepared: CLC1 $=65 \%$ BL090 $+35 \%\{89 \% \mathrm{SB}+11 \% \mathrm{RMB}\}$ and CLC2 $=70 \%$ BL090 $+30 \%\{87.2 \% \mathrm{SB}+12.8 \% \mathrm{RMB}\}$. The variation of concentration permits the changing and the tuning of the pitch inside the near-IR range. For each blend the concentration in photocrosslinkable material is about $3.8 \%$.

$12.5 \mu \mathrm{m}$ thick cells are made with ITO glass plates. In order to induce a planar orientation, plates were treated with polyvinyl alcohol (PVA 15'000, ref. 81381 from Fluka) in water solution (at $1 \mathrm{wt} . \%$ ). The mixtures are introduced in cells at $120^{\circ} \mathrm{C}$.

Each UV-curing sequence is carried out during 20 minutes at $365 \mathrm{~nm}$ with a power of $0.1 \mathrm{~mW} . \mathrm{cm}^{-2}$ measured with an UV-radiometer (UVR-365 from Prolabo).

The plates are split when the cell is put in contact with liquid nitrogen in order to avoid plastic disturbances.

Spectral characteristics are obtained by unpolarized UV-visible-near IR spectrophotometry (UV-3100 Shimadzu) in transmittance mode and at ambient temperature. It is checked that negative peaks are due to reflection and not to absorbance. The baseline was done when a couple of ITO glass substrates treated with PVA were put in each arm. It permits the normalization of the transmittance of sandwich-cells since the level of light transmittance for ITO glass in the wavelength range of the present investigation $(1.0-2.6 \mu \mathrm{m})$ is not constant.

\section{RESULTS AND DISCUSSION}

\section{A. Reflection properties: from individual films to the sandwich-cell}

PSCLC1 and PSCLC2 cells are CLC1 and CLC2 cells after UV-curing. Why use PSLCs in the present study? Generally, the basic idea of introducing a polymer 
network into the LC matrix is to transfer the orientational order of the mesophase to the macromolecular architecture during the polymerization and crosslinking [27]. The original texture is thus stabilized by the introduction of a large surface to volume ratio as compared to a simple orientation by substrate alignment. Here, in order to elaborate a sandwich-cell with PSLCs instead of LCs we also researched to let diffusion between chemical species to occur but by limiting it due to the action of polymer network which preserves some structural properties of the mesophase during the UV-curing. Indeed, the trivial situation of an homogeneous mixture with a pitch intermediate between the two individual pitches would not be desirable.

The cells are then opened and the substrates with the films are kept. A $12.5 \mu \mathrm{m}$ thick sandwich-cell is made with the two semi-free films and UV-irradiated. Why this supplementary UV-curing sequence after the cell has been closed? The goal is to polymerize and to crosslink the material which had not reacted during the previous individual sequences. Two effects are expected from this mode of operating: $(i)$ to insure a dielectric continuity at the interface between films since the cell is devoted to becoming electric-field switchable; (ii) to insure a continuity in the optical properties since the appearance of a demarcation between transmittance spectra intrinsic to pristine films is not wanted; the effect of this final UV-curing on the optical properties after the cell has been closed will be analyzed in section B.

Figure 1 shows the variation of transmitted light with wavelength for PSCLC1 \& 2 semi-free films and the sandwich-cell; let us mention that the slight increase of transmittance when the wavelength increases is a consequence of spectra normalization as indicated at the end of the Experimental Section III. The spectra of individual films are not significantly altered by the opening of cells at low temperature as described in section III. The spectrum inherent to the 'bilayer'-cell is not entirely the 
sum of the individual spectra related to the initial films. First, the spectrum is continuous: there is no clear discontinuity which might represent a frontier between the layers; this result is important in terms of optical performances. Then, the wavelength band $\Delta \lambda$ for reflection - measured as usually at the half-height of the peak - is about $460 \mathrm{~nm}$. As an indicator, it is crucial to compare this value to which is usually encountered for cholesteric reflections in the related part of the near-IR spectrum. Figure 2 shows the variation of $\Delta \lambda$ with $\lambda$ from about 1650 to $2200 \mathrm{~nm}$ in the present three-component mixture when BL090 concentration varies from 63 to $70 \%$. At $1810 \mathrm{~nm}$ - which is the mean wavelength affected to the peak of the sandwich-cell $-\Delta \lambda$ is about $310 \mathrm{~nm}$. Therefore, the reflection band is $50 \%$ greater than the band obtained at comparable mean wavelengths for related CLCs.

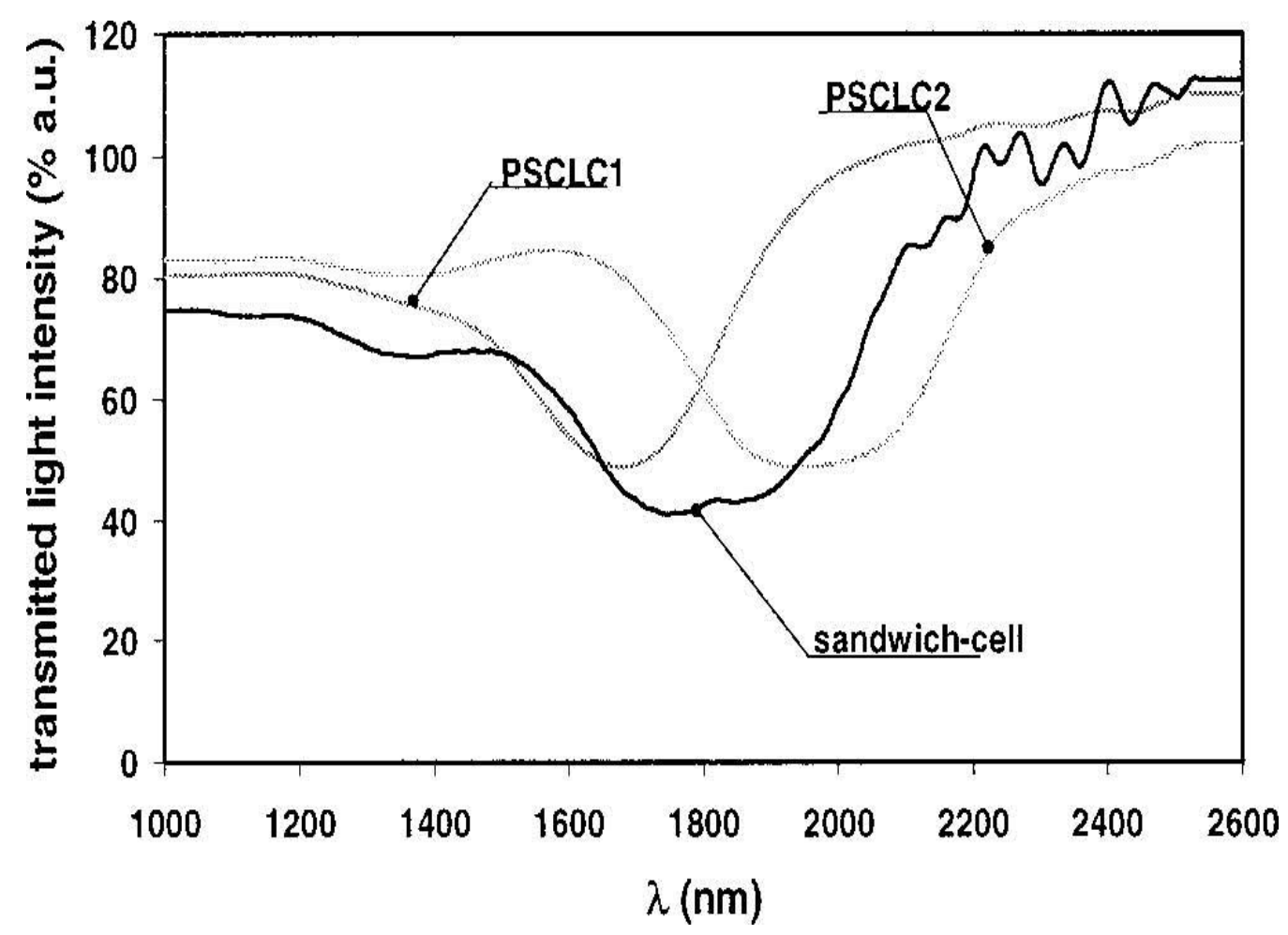

FIG. 1. Transmitted light intensity as a function of wavelength $\lambda$ for the individual semifree films PSCLC1 and PSCLC2 and for the UV-cured sandwich-cell made with them. 


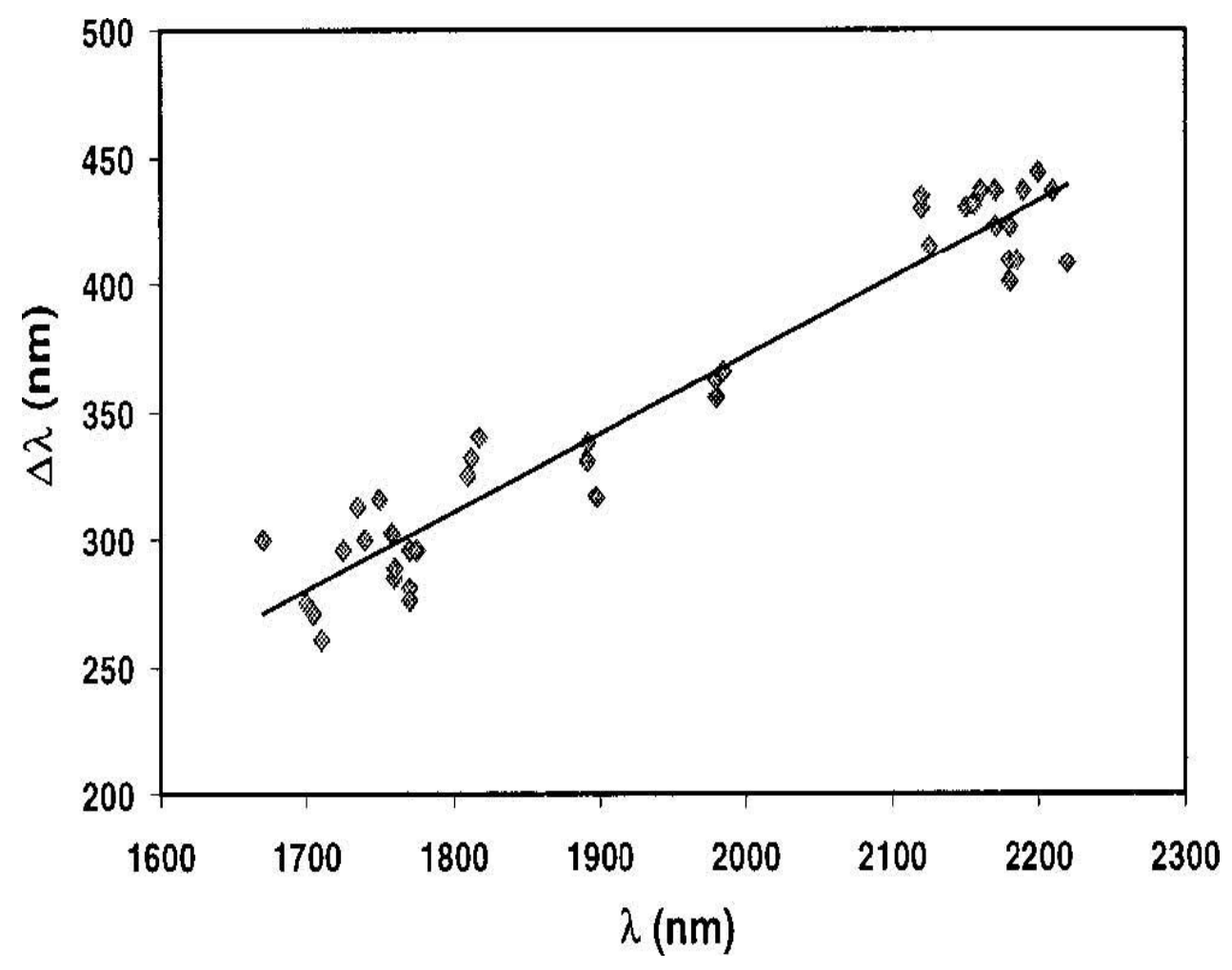

FIG. 2. Reflection wavelength band $\Delta \lambda$ vs. wavelength $\lambda$ for the three-component cholesteric mixture RMB, SB and BL090 when BL090 concentration varies from 63 to $70 \%$. The straight line (linear fit) is a guide for the eye.

\section{B. Time stability}

The sandwich-cell intrinsically involves a contact between two CLC gels. The morphology of such films is a porous macromolecular network trapping low molecular mass LCs. As a consequence, low molecular mass LCs, as well as a fraction of incompletely crosslinked polymeric structures, may diffuse between layers. It was thus relevant to investigate the time stability of optical properties. Figure 3 represents the 
variation in the final cell of the mean reflection wavelength $\lambda$ and the bandwidth $\Delta \lambda$ with time.
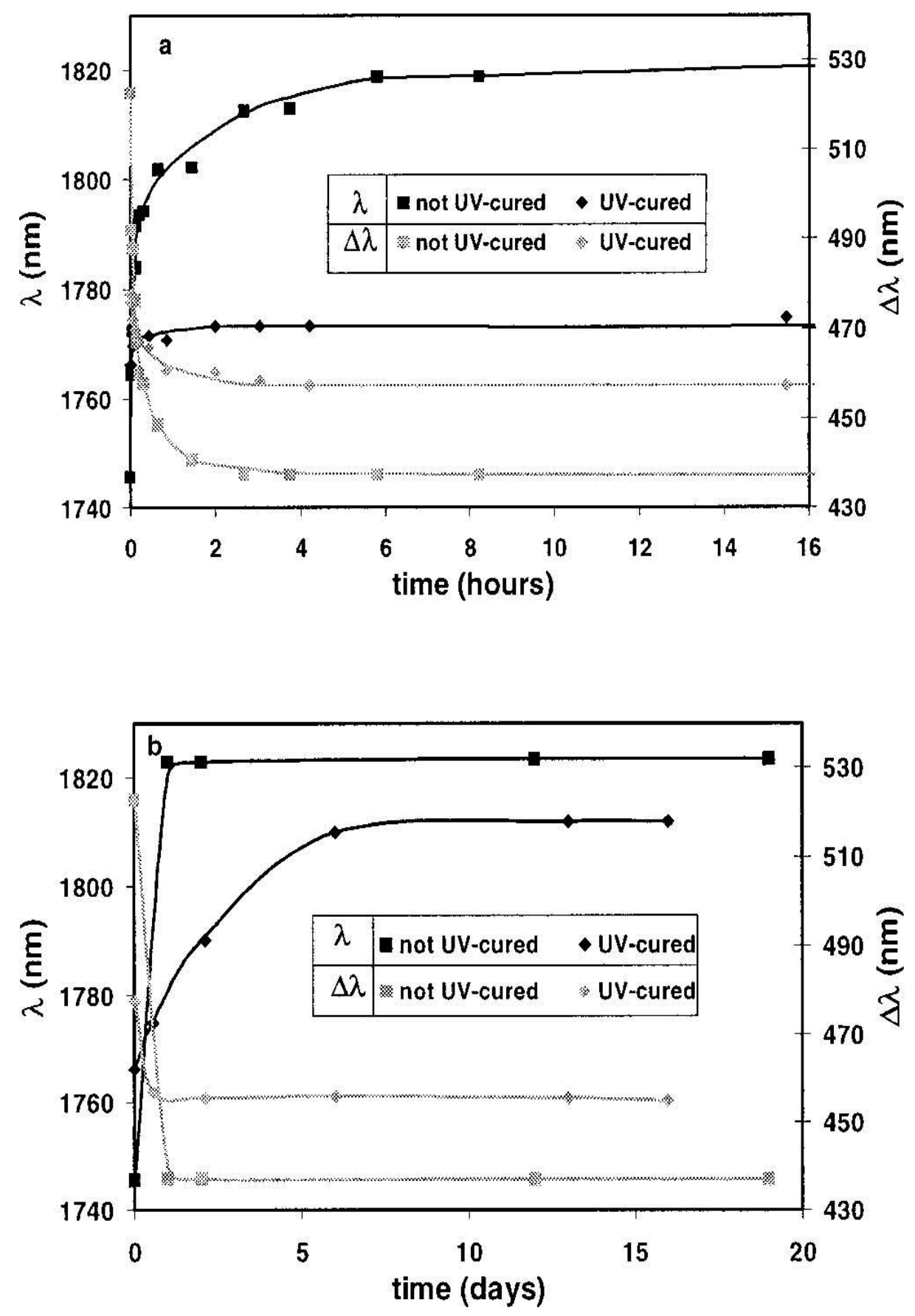

FIG. 3. Wavelength $\lambda$ and reflection band $\Delta \lambda$ for the sandwich-cell as a function of time: (a) on a 16-hour period; (b) on a 20-day period. The cases for which the cell is UV-cured after sealing or not are distinguished. 
To discuss the role of the UV-curing after the two layers are put in contact, data corresponding to the case where the sandwich-cell is not UV-irradiated are also displayed. For presentation clarity, results are divided into two groups: (a) 'short'-time stability (up to 16 hours, Figure 3.a) and (b) 'long'-time stability (on a 20-day period, Figure 3.b). Comments may be summarized as follows: (1) On $\lambda$ : if the cell is not UV-cured after the layers are put in contact, $\lambda$ is progressively shifted towards greater wavelengths - the shift is about $80 \mathrm{~nm}$ - until stabilization occurs above 6 hours. The system reaches an equilibrium concentration and thus a global apparent pitch which is shifted towards the greatest pitch value of the two layers. When the cell is UV-cured, the phenomenon of drift in the position of $\lambda$ is still present but less pronounced - about $45 \mathrm{~nm}$. Another difference lies in the kinetics of such a drift which are visible on a 6-day period (Figure 3.b). The variation of $\lambda$ with time is soft, probably due to the fact than diffusion here is occurring in a medium with a more important degree of polymerization and crosslinking compared to the cell without supplementary irradiation. Diffusion of molecules unbound to the macromolecular network is slowed down, and also restricted as a consequence of a stabilization-effect brought by the final UV-curing. (2) On bandwidth $\Delta \lambda$ : without UV-curing, $\Delta \lambda$ decreases by about $85 \mathrm{~nm}$. After UV-curing, and probably with the same kind of stabilization effect as described for $\lambda, \Delta \lambda$ decrease is now limited to $20 \mathrm{~nm}$ and $\Delta \lambda$ is maintained close to $460 \mathrm{~nm}$. Significant exchanges between layers - as a result of interdiffusion - which modify $\Delta \lambda$ happen on a one-day period. A subsequent UV-curing of the sandwich-cell acts to limit the concentration distributions between layers which would reduce $\Delta \lambda$. 


\section{Electro-optical behaviour}

Figure 4 shows an example of three typical states which may be reached when a voltage is applied to the cell; the inserted cross-sectional views of cells are purely tentative and do not predict the exact morphology of films. At zero or very weak voltages (e.g.: $0.86 \mathrm{~V}$ ), the cell exhibits a broadband reflection. Then the position as well as the bandwidth of reflection peak are modified and the system goes from a reflecting to a scattering state (e.g.: 47.0 V). As in PSCLCs with a narrow band for reflection, light scattering is due to a focal conic texture exhibiting polydomains [28]. This is the result of a competition between LC molecules close to the polymer network which have a tendency to contribute to a stable planar reflecting texture and the electric field effect which is to destroy this order by untwisting the helix. Finally and for higher voltages, the cell becomes transparent (e.g.: $169 \mathrm{~V}$ ) when the structure is untwisted and free LC molecules are perpendicular to the electrodes.

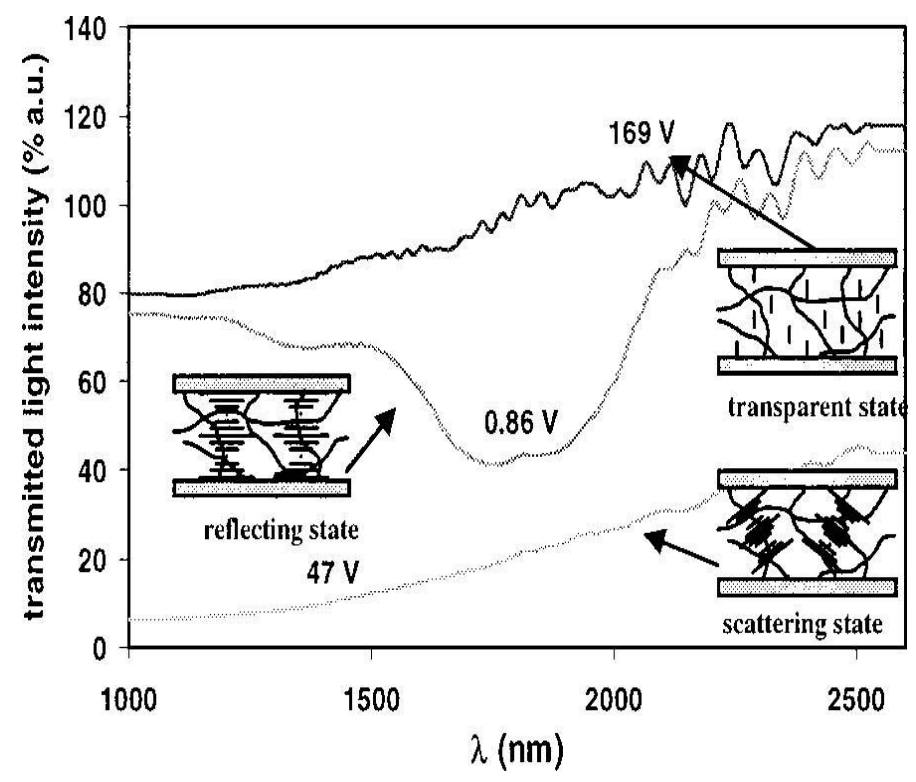

FIG. 4. Transmitted light intensity vs. wavelength $\lambda$ for the UV-cured sandwich-cell when three different typical voltages are applied. Inserted: schematic drawings of the 
PSLC material, i.e. a polymer network embedding rod-like LC free molecules with a twisted or an untwisted (nematic-like) order. These schemes are purely tentative and do not predict the exact morphology of films.

As a three-dimensional view, Figure 5 shows the continuous evolution of transmitted light intensity with wavelength and voltage. Two events are especially typical of the history of light transmittance, save the transparency obtained at high voltages: $(i)$ the reflecting mode at low voltages and in the middle of the wavelength range; (ii) and the scattering mode between $35-40$ and $115-140$ V which is weakly wavelength-dependent in the expected range.

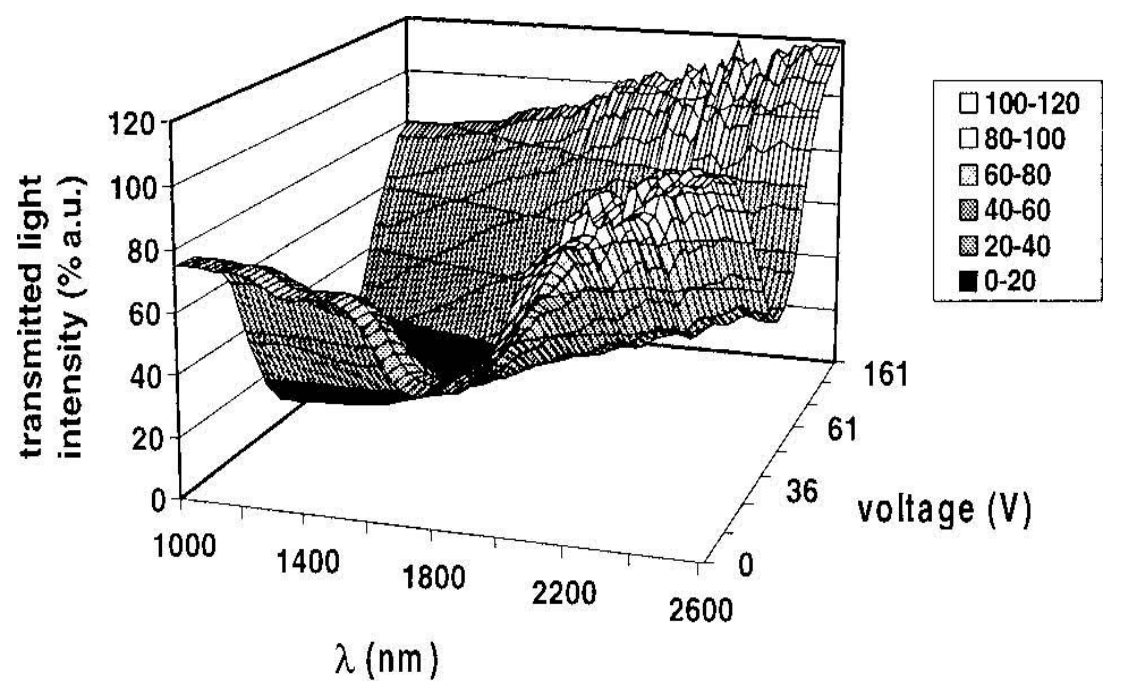

FIG. 5. 3-D representation of transmitted light intensity vs. wavelength $\lambda$ and voltage for the UV-cured sandwich-cell. 


\section{CONCLUSIONS}

We have presented new PSCLCs with a light reflection band in the near-IR spectrum broader than usually expected at comparable wavelengths. These materials are the association of two individual PSCLC layers for whom the interdiffusion is controlled by UV-curing which acts to preserve the bandwidth value. The material design is independent of the wavelength range which is chosen. Stability of systems with time was investigated and the reflection bandwidth was stabilized on a one-day period. The optical characteristics can be tuned by applying an electric field and electro-optical transitions between broadband reflecting, scattering and transparent states are made possible.

Switchable broadband reflections in the IR spectrum are relevant for 'smart' windows in buildings when a control over the solar spectrum, without color effects, is desirable. By choosing cholesteric pitch values in the visible spectrum, this method might potentially be interesting for the manufacture of white-or-black free-polarizer screens with high brightness.

\section{ACKNOWLEDGMENTS}

The authors are grateful to Drs. F.-H. Kreuzer and E. Hanelt (Wacker-Chemie $\mathrm{GmbH}$, Germany) for providing RMB and SB. This work was supported in part by The European Commission under the project Joule III/SMARTWIN, CT97-0068. We thank Matthew Hart for his assistance in English. 
[1] de Gennes, P.G., and Prost, J., 1993, The Physics of Liquid Crystals (Oxford: Clarendon Press), pp. 263-280.

[2] For a recent review: Boiko, N., and Shibaev, V., 2000, Intern. J. Polymeric. Mater., 45, 533.

[3] Broer, D.J., Lub, J., and Mol, G.N, 1995, Nature, 378, 467.

[4] For a review: Broer, D.J., Mol, G.N., van Haaren, A.M.M., and Lub, J., 1999, Adv. Mater., 11, 7, 573.

[5] Coates, D., Goulding, M.J., Greenfield, S., Hanmer, J.M.W., Marden, S.A., and Parri, O.L., Proc. SID, 1996, 67.

[6] Coates, D., Goulding, M.J., Greenfield, S., Hanmer, J.M.W., Marden, S.A., Parri, O.L., Verrall, M., and Ward, J., Proc. IDW, 1996, 309.

[7] Guillard, H., Sixou, P., and Gandolfo, V., Project SMARTWIN, Joule III Programme, CT97-0068, The European Commission, Mid-term report, june 1999.

[8] Schlichting, W., Faris, S., Li, L., Fan, B., and Kralik, J., Proc. SPIE, 1996, 2690, 232.

[9] Schlichting, W., Faris, S., Li, L., Fan, B., Kralik, J., Haag, J., and Lu, Z., 1997, Mol. Cryst. Liq. Cryst., 301, 231.

[10] Lu, Z., Li, L., Vithana, H., Jiang, Y., and Faris, S.M., 1997, Mol. Cryst. Liq. Cryst., 301, 237.

[11] Li, L., and Faris, S.M., Proc. SID, 1996, 111.

[12] Kralik, J.C., Fan, B., Vithana, H., Li, L., and Faris, S.M., 1997, Mol. Cryst. Liq. Cryst., 301, 249, p. 251.

[13] Schlichting, W., Faris, S., Fan, B., Haag, J., Lu, Z., Kane, S., Li, L., Milster, T., and Luo, H., 1997, Jap. J. of Appl. Phys., 36, 587.

[14] Mitov, M., Boudet, A., and Sopéna, P., Project SMARTWIN, Joule III Programme, CT97-0068, The European Commission, First periodic progress report, june 1998. 
[15] Mitov, M., Boudet, A., and Sopéna, P., 1999, Eur. Phys. J. B, 8, 327.

[16] Binet, C., Mitov, M., and Boudet, A., 2000, Mol. Cryst. Liq. Cryst., 339, 111.

[17] Boudet, A., Binet, C., Mitov, M., Bourgerette, C., and Boucher, E., 2000, Eur. Phys. J. E, 2, 247.

[18] Sixou, P., Guillard, H., and Gautier, C., $18^{\text {th }}$ International Liquid Crystal Conference, Sendai, Japan, 2000, poster 25D-7-P.

[19] Chen, S.H., Jin, R.J., Katsis, D., Mastrangelo, J.C., Papernov, S., and Schmid, A.W., 2000, Liq. Cryst., 27, 201.

[20] Lavernhe, A., Mitov, M., Binet, C. and Bourgerette, C., 2001, Liq. Cryst., 28, 803.

[21] Leroux, N., and Chien, L.-C., 1996, Proc. of the ACS Meeting, New-Orleans, Vol. $37, \mathrm{n}^{\circ} 1,195$.

[22] Leroux, N., Fritz, W.J., Doane, J.W., and Chien, L.-C., 1995, Mol. Cryst. Liq. Cryst., 261, 465.

[23] Dyer, D.J., Schröder, U.P., Chan, K.-P., and Twieg, R.J., 1997, Chem. Mater., 9, 1665.

[24] (a) Hikmet, R.A.M., and Kemperman, H., 1998, Nature, 392, 476. (b) Hikmet, R.A.M., and Kemperman, H., 1999, Liq. Cryst., 26, 1645.

[25] Binet, C., Mitov, M., and Mauzac, M., $18^{\text {th }}$ International Liquid Crystal Conference, Sendai, Japan, 2000, poster 25E-22-P.

[26] Kreuzer, F.H., Andrejewski, D., Haas, W., Häberle, N., Riepl, G., and Spes, P., 1991, Mol. Cryst. Liq. Cryst., 199, 345.

[27] For a recent review: Dierking, I., 2000, Adv. Mater., 12 (3), 167.

[28] Yang, D.-K., Chien, L.-C., Doane, J.W., 1992, Appl. Phys. Lett., 60 (25), 3102. 